\title{
Research on thrust vector control of nonlinear solid rocket motor nozzle based on active disturbance rejection technology
}

\author{
Chenhong Shi ${ }^{1, *}$, Jinpeng Yang ${ }^{1}$, and Zhishu $\mathrm{Xu}^{1}$ \\ ${ }^{1}$ Beijing institute of precision electromechanical control equipment, Beijing, 100076, China
}

Keywords: Auto disturbance rejection control technology, Non-linear load, Rocket nozzle, Thrust vector control.

\begin{abstract}
In this paper, based on the auto disturbance rejection control technology, the electromechanical servo system is used as the control actuator, and the thrust vector control of the solid rocket motor nozzle with typical nonlinear friction characteristics is studied and analyzed. In this paper, the realization of the classical PID algorithm and the lack of dynamic performance are analyzed, and then the compensation algorithm based on the auto disturbance rejection control technology is added. The algorithm compensates for the phase lag of the system due to the nonlinear friction characteristics. As a result, the frequency characteristics of the system have been significantly improved.
\end{abstract}

\section{Introduction}

The launch vehicle technology using solid rocket motors has made great progress [1]-[3]. The thrust vector control using solid rocket motor nozzles with full-axis swing has been widely used due to its many advantages such as small thrust loss and large control torque[4]. At present, this technology has become a trend of technology development.

The full-axis swing nozzle of a typical solid rocket motor includes different types of flexible nozzle, bead nozzle, ball and socket nozzle, and the bead nozzles and the ball and socket nozzles are mainly characterized by typical nonlinear friction load characteristics. The classical PID control algorithm can achieve stable control of the bead nozzle and the ball and socket nozzle, but it is difficult to solve the influence of the inherent nonlinear friction load characteristics of the nozzle on the control characteristics, especially the phase lag generated. In this paper, the electromechanical servo system is used as the control actuator, and the control compensation algorithm based on the auto disturbance rejection control technology is used to improve the thrust vector control performance of the nonlinear nozzle system[5]-[7].

\footnotetext{
* Corresponding author: yjpeng620@163.com
} 


\section{Nonlinear load characteristics of solid rocket motor nozzle}

The widely used bead nozzle and ball and socket nozzle of solid rockets are mechanical, full-axis swing solid rocket motor nozzles. The bead nozzle supports the injection load by one or more rows of balls between the movable convex spherical surface and the immovable concave spherical surface; and the ball and socket nozzle cancels the balls in the bead nozzle, so that the convex spherical surface and the concave spherical surface direct contact. The relative motion between the convex and concave surfaces of the two nozzles achieves a full-axis swing of the nozzle.

Through the study of the nozzle load characteristics, the main load of the bead nozzle is the contact motion friction torque of the balls contact with the point of the convex spherical surface and the concave spherical surface; the main load of the ball and socket nozzle is the frictional moment of the direct surface contact movement between the convex spherical surface and the concave spherical surface. Although there are differences in the structure of the two nozzles, there are differences in the mechanism of the friction torque, but the load torque characteristics are the friction load torque. At the same time, due to the complexity of the friction characteristics, they all exhibit obvious nonlinear characteristics[8]- [10]. The simplified load model is shown in Figure 1.

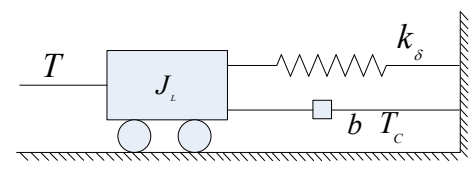

Fig. 1. Simplified nonlinear load model.

In the figure: $T$ - The torque of the servo mechanism;

$J_{L}$ - The moment of inertia of the nozzle;

$b$ - The viscous friction coefficient of the nozzle;

$k_{\delta}$ - Elastic load torque gradient of the nozzle;

$T_{c}$ - Coulomb friction torque of the nozzle.

According to the actual design of the project, the elastic load torque of the bead nozzle and the ball and socket nozzle itself is very small, and the elastic torque only provides the necessary torsion resistance torque, so the elastic load torque gradient is usually negligible. Therefore, the leading role is the frictional moment opposite to the direction of the nozzle swinging speed. The complex friction torque load characteristics can be further simplified to the viscous frictional torque associated with the nozzle swing velocity vector and the Coulomb frictional torque associated only with the direction of the swing velocity. In engineering, the viscous friction torque can be approximated as increasing with the increase of the swing speed, and the relationship between the magnitude of the moment and the swing speed is characterized by the viscous friction coefficient. The Coulomb friction torque is only opposite to the direction of the swing speed. Its size is approximately fixed in engineering. It is a typical nonlinear load torque, which is also a concrete manifestation of the nonlinear load characteristics of the bead nozzle and the ball and socket nozzle.

For the stability design of the attitude control system, the desired control amount is the angular displacement output of the nozzle, and the actual servo control can only control the linear displacement output of the actuator to the closed loop. As shown in picture 2 . 


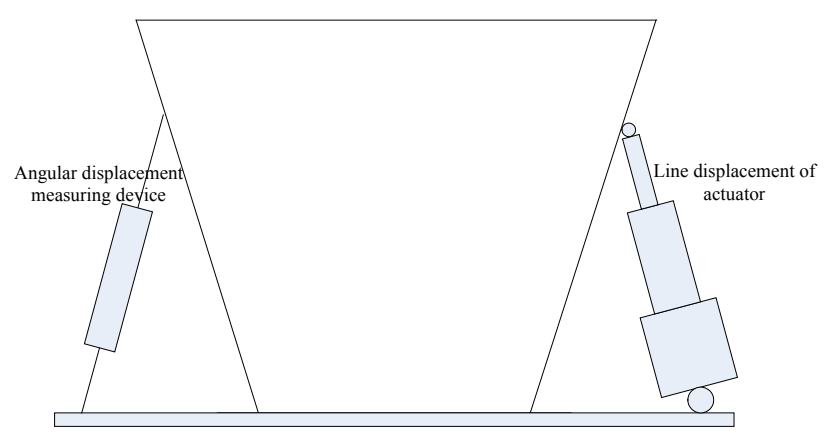

Fig. 2. Line displacement and angular displacement of thrust vector control.

Since the transmission process from line displacement to angular displacement is the open-loop process, and the nozzle itself has significant Coulomb friction nonlinear link, and the structural stiffness of the nozzle itself cannot be approximated to infinity due to structural quality constraints and other factors. Therefore, there must be a significant difference between the linear displacement and the angular displacement, which is the inherent characteristic of a solid rocket motor nozzle with nonlinear friction load characteristics.

\section{Nonlinear load characteristics of solid rocket motor nozzle comparative analysis of classic PID control and ADRC}

\subsection{Classic PID control strategy}

As an actuator, electromechanical servo system has been widely used in various thrust vector control systems. There are many adjustment methods for electromechanical servo system, and the design of correction control network is flexible. With the classic PID control strategy, the "torque-speed-position" three-loop closed-loop control can be conveniently realized. Through the closed-loop control, the thrust vector control of the solid rocket motor nozzle such as the bead nozzle or the ball and socket nozzle can be reliably realized.

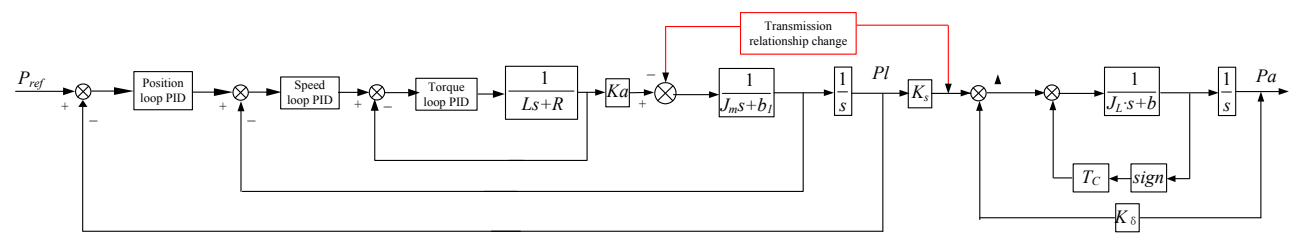

Fig. 3. Solid motor nozzle thrust vector control block diagram based on electromechanical servo system.

In the figure:

$$
\begin{array}{lll}
P_{r e f} & -- & \text { System control instruction; } \\
L & -- & \text { Servo motor inductance; } \\
R & -- & \text { Servo motor resistance; } \\
K_{a} & -- & \text { Servo motor torque coefficient; } \\
J_{m} & -- & \text { The moment of inertia of the electromechanical actuator is } \\
& & \text { equivalent to the motor side; }
\end{array}
$$


$b_{1} \quad--\quad$ Equivalent viscous friction coefficient of electromechanical transmission mechanism;

$\mathrm{Pl} \quad$-- $\quad$ Actuator line displacement output;

$K_{s} \quad-\quad$ Motor nozzle structure support stiffness;

sign -- Take the direction symbol;

$\mathrm{Pa} \quad$-- Nozzle angular displacement output.

Since the electromechanical servo system can achieve a large forward gain, the line displacement can better follow the system control command under typical position commands. However, since the nonlinear Coulomb friction torque of the bead nozzle or the ball and socket nozzle itself is large, and is limited by the quality of the nozzle structure, the support stiffness value of the nozzle itself may not be large. Therefore, there must be a significant difference between the angular displacement and the linear displacement.

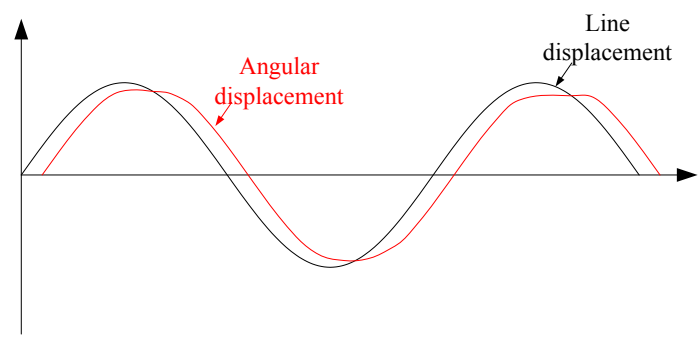

Fig. 4. Difference between displacement and angular displacement of thrust vector control line under nonlinear load conditions.

\subsection{Compensation control of nonlinear characteristics by active disturbance rejection control algorithm}

In a control system that does not require high control accuracy and dynamic characteristics, the difference between line displacement and angular displacement in Figure 4 does not have a substantial impact on the control stability of the rocket flight. However, with the continuous improvement of the flight performance of the rocket, especially in the first-class flight of the rocket, there are often design contradictions such as large flying dynamic pressure, high static instability of the rocket and low elastic mode frequency. Therefore, the requirements for the angular displacement performance index are continuously improved, and the design goal is that the angular displacement output in the middle and low frequency bands is as close as possible to the control command input.

Due to the inherent existence of the nonlinear characteristics of the nozzle, the PID control algorithm based on the classical control theory cannot solve the amplitude attenuation and phase lag caused by the nonlinear link. The root cause of amplitude attenuation and phase lag is that the inherent nonlinear Coulomb friction torque $\left(T_{c}\right)$ of the nozzle is large, while the support stiffness $\left(K_{s}\right)$ of the nozzle is relatively low. The larger of the value of $\frac{T_{c}}{K_{s}}$, the larger the difference between the linear displacement and the angular displacement, and the worse the following characteristic of the control; otherwise, the difference between the linear displacement and the angular displacement is small, and the control following characteristic is better. Since the nozzle has a relatively large dispersion of the Coulomb friction torque during the complicated process production process, the Coulomb friction will change accordingly due to the change of the internal pressure of the solid rocket motor during different working periods. Therefore, although the support 
stiffness of the nozzle can be determined as a constant value by the test, the control strategy of the fixed value compensation cannot be adopted due to the large dispersion of the Coulomb friction torque. Therefore, the Coulomb friction torque value must be obtained by online real-time estimation method, and on this basis, the control compensation is completed.

Real-time compensation control of solid rocket motor nozzles with non-linear load characteristics can be realized by using an integral expansion state observer based on auto disturbance rejection control technology[11]- [13]. Since the control of the electromechanical servo system is based on the servo motor, the relevant parameter variables are equivalent to the rotor side of the servo motor. The compensation algorithm is designed according to the dynamic balance equation of the servo motor torque:

$$
T_{M}=J_{M} \cdot \ddot{\theta}+b_{M} \cdot \dot{\theta}+K_{M} \cdot \theta+\operatorname{sign}(\dot{\theta}) \cdot T_{\text {coulomb }}
$$

In the equation:
$T_{m}$
-- The output torque of the servo motor;
$J_{m} \quad$-- $\quad$ The system is equivalent to the moment of inertia on the servo motor side;
$b_{m} \quad$-- The system is equivalent to the viscous friction coefficient of the servo motor side;
$K_{m} \quad$-- $\quad$ The system is equivalent to the elastic moment gradient on the servo motor side;
$T_{\text {coulomb }} \quad$-- $\quad$ The system is equivalent to the Coulomb friction load torque on the servo motor side;
$\theta \quad--\quad$ The swing angle of the solid rocket motor nozzle;
$\dot{\theta} \quad--\quad$ Swing angular velocity of a solid rocket motor nozzle;
$\ddot{\theta} \quad$-- The angular acceleration of the solid rocket motor nozzle.

In order to facilitate the analysis using the method of active disturbance rejection technology, the above equation is transformed into a form that is easy to process in state space:

$$
\ddot{\theta}=-\frac{b_{M}}{J_{M}} \cdot \dot{\theta}-\frac{K_{M}}{J_{M}} \cdot \theta-\frac{\operatorname{sign}(\dot{\theta})}{J_{M}} \cdot T_{\text {coulomb }}+\frac{T_{M}}{J_{M}}
$$

Let $x_{1}=\theta, x_{2}=\dot{\theta}$, and derive the state space equation of the form:

$$
\left\{\begin{array}{l}
\dot{x}_{1}=x_{2} \\
\dot{x_{2}}=-\frac{b_{M}}{J_{M}} \cdot x_{2}-\frac{K_{M}}{J_{M}} \cdot x_{1}-\frac{\operatorname{sign}\left(x_{2}\right)}{J_{M}} \cdot T_{\text {coulomb }}+\frac{T_{M}}{J_{M}} \\
y=x_{1}
\end{array}\right.
$$

The above state observer can implement the tracking of the variables in the system, estimate the $x_{1}$ by $Z_{1}$, estimate $x_{2}$ with $Z_{2}$, estimate the interference term $\left(-\frac{\operatorname{sign}\left(x_{2}\right)}{J_{M}} \cdot T_{\text {coulomb }}\right)$ with $Z_{3}$, and define: 


$$
\left\{\begin{array}{l}
e=Z_{1}-y \\
L_{1}=3 \cdot \omega_{0} \\
L_{2}=3 \cdot \omega_{0}^{2} \\
L_{3}=\omega_{0}^{3}
\end{array}\right.
$$

$\omega_{0}$ is the parameter to be designed. According to the relevant parameter values of the system and the characteristic parameter range of the load, a reasonable value is set by the combination of simulation and test. Therefore:

$$
\left\{\begin{array}{l}
\dot{Z}_{1}=Z_{2}-L_{1} \cdot e \\
\dot{Z}_{2}=-\frac{b_{M}}{J_{M}} \cdot Z_{2}-\frac{K_{M}}{J_{M}} \cdot Z_{1}+Z_{3}+\frac{T_{M}}{J_{M}}-L_{2} \cdot e \\
\dot{Z}_{3}=-L_{3} \cdot e
\end{array}\right.
$$

In the specific implementation of the control algorithm, the DSP processor is used to implement the active disturbance rejection control algorithm using the digital discrete control method. Let $h$ be the closed-loop time period of the discrete system, then:

$$
Z_{1}=Z_{1-1}+h \cdot \dot{Z}_{1}
$$

where $Z_{1-1}$ is the saved value of $Z_{1}$ 's last discrete time period, and the same is true:

$$
\left\{\begin{array}{l}
Z_{1}=Z_{1-1}+h \cdot\left(Z_{2-1}-L_{1} \cdot e\right) \\
Z_{2}=Z_{2-1}+h \cdot\left(-\frac{b_{M}}{J_{M}} \cdot Z_{2-1}-\frac{K_{M}}{J_{M}} \cdot Z_{1-1}+Z_{3-1}+\frac{T_{M}}{J_{M}}-L_{2} \cdot e\right) \\
Z_{3}=Z_{3-1}+h \cdot\left(-L_{3} \cdot e\right)
\end{array}\right.
$$

For the bead nozzle or the ball and socket nozzle, since the elastic load torque can be neglected, it is considered that $K_{M}=0$, thereby simplifying the above formula:

$$
\left\{\begin{array}{l}
Z_{1}=Z_{1-1}+h \cdot\left(Z_{2-1}-L_{1} \cdot e\right) \\
Z_{2}=Z_{2-1}+h \cdot\left(-\frac{b_{M}}{J_{M}} \cdot Z_{2-1}+Z_{3-1}+\frac{T_{M}}{J_{M}}-L_{2} \cdot e\right) \\
Z_{3}=Z_{3-1}+h \cdot\left(-L_{3} \cdot e\right)
\end{array}\right.
$$

From this, it is possible to estimate the Coulomb friction load torque $\left(T_{\text {coulomb }}\right)$ of the system equivalent to the servo motor side.

$$
T_{\text {coulomb }}=-Z_{3} \cdot \frac{J_{M}}{\operatorname{sign}\left(x_{2}\right)}=-\operatorname{sign}\left(x_{2}\right) \cdot J_{M} \cdot Z_{3}
$$

Finally, the control compensation amount can be directly calculated by using the nozzle support stiffness $\left(K_{s M}\right)$ determined by the test to the servo motor side:

$$
\Delta \theta=\frac{T_{\text {coulomb }}}{K_{s M}}=\frac{-\operatorname{sign}\left(x_{2}\right) \cdot J_{M} \cdot Z_{3}}{K_{s M}}
$$

Positive feedback of the compensation amount $(\Delta \theta)$ into the control command signal can partially offset the deformation effect of the nozzle under the nonlinear Coulomb friction 
torque, thus improving the amplitude and phase of the signal following. The system block diagram added with the auto disturbance rejection control algorithm is shown in the figure below.

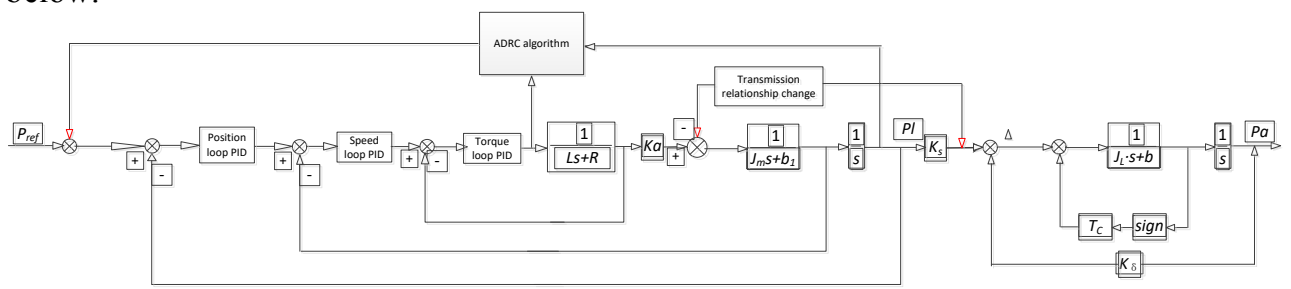

Fig. 5. System block diagram with automatic disturbance rejection control algorithm.

The compensated system line displacement and angular displacement are as follows.

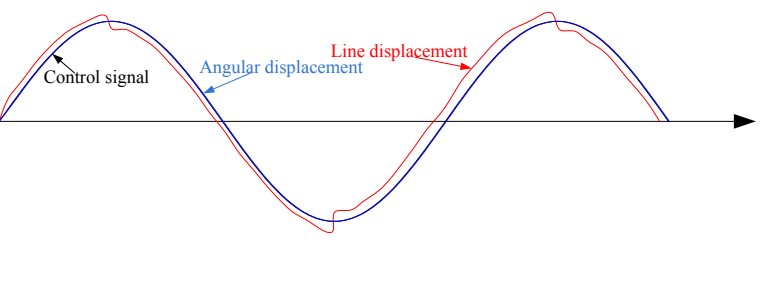

Fig. 6. Linear displacement and angular displacement after compensation by the active disturbance rejection algorithm.

Under the real load condition of a certain type of ball and socket nozzle, the frequency characteristic data of the classical PID control algorithm and the auto disturbance rejection control algorithm are compared as shown in the following table. The Bode diagram is compared as shown in the following figure.

Table 1. Comparison of frequency characteristic data.

\begin{tabular}{|c|c|c|c|c|c|c|c|c|}
\hline \multirow{3}{*}{$\omega$} & \multicolumn{4}{|c|}{ No compensation algorithm } & \multicolumn{3}{c|}{ Add active disturbance rejection control algorithm } \\
\cline { 2 - 9 } & Line displacement & Angular displacement & \multicolumn{2}{c|}{ Line displacement } & \multicolumn{2}{c|}{ Angular displacement } \\
\cline { 2 - 9 } & Amp & Phase & Amp & Phase & Amp & Phase & Amp & Phase \\
\hline 1 & -1.46 & -6.17 & -5.24 & -30.44 & 0.725 & 3.968 & -1.608 & -16.02 \\
\hline 2 & -1.32 & -7.10 & -4.35 & -32.48 & 0.924 & 4.04 & -1.141 & -15.28 \\
\hline 6.28 & -1.45 & -8.48 & -4.82 & -35.94 & 1.233 & -2.775 & -1.201 & -21.853 \\
\hline 10 & -1.55 & -9.97 & -5.09 & -39.75 & 1.276 & -8.744 & -1.217 & -29.256 \\
\hline 16 & -1.64 & -11.89 & -5.41 & -43.02 & 1.088 & -16.81 & -1.378 & -38.959 \\
\hline 20 & -1.67 & -13.21 & -5.42 & -45.23 & 0.838 & -21.26 & -1.503 & -44.410 \\
\hline 28 & -1.76 & -15.61 & -5.72 & -48.41 & -0.211 & -28.29 & -2.975 & -54.875 \\
\hline 40 & -1.74 & -20.81 & -4.52 & -55.40 & -0.975 & -32.19 & -3.067 & -62.656 \\
\hline 60 & -1.72 & -30.46 & -2.52 & -68.57 & -1.427 & -40.71 & -1.957 & -75.749 \\
\hline 100 & -2.56 & -41.68 & -3.10 & -86.97 & -2.647 & -50.18 & -3.195 & -93.885 \\
\hline 120 & -2.87 & -48.71 & -2.39 & -96.38 & -2.956 & -57.46 & -2.656 & -103.65 \\
\hline 160 & -4.98 & -58.12 & -3.21 & -135.1 & -4.983 & -68.03 & -3.388 & -142.66 \\
\hline 180 & -5.07 & -57.11 & -5.58 & -149.8 & -5.075 & -68.12 & -5.859 & -158.12 \\
\hline 200 & -4.88 & -58.11 & -7.63 & -158.5 & -4.886 & -70.30 & -7.924 & -167.75 \\
\hline 220 & -4.64 & -62.26 & -10.22 & -166.2 & -4.63 & -75.55 & -10.36 & -175.00 \\
\hline 240 & -4.51 & -77.81 & -11.64 & -183.6 & -4.528 & -79.01 & -11.73 & -178.43 \\
\hline 260 & -4.30 & -85.33 & -14.10 & -186.9 & -4.315 & -88.63 & -14.57 & -183.06 \\
\hline
\end{tabular}



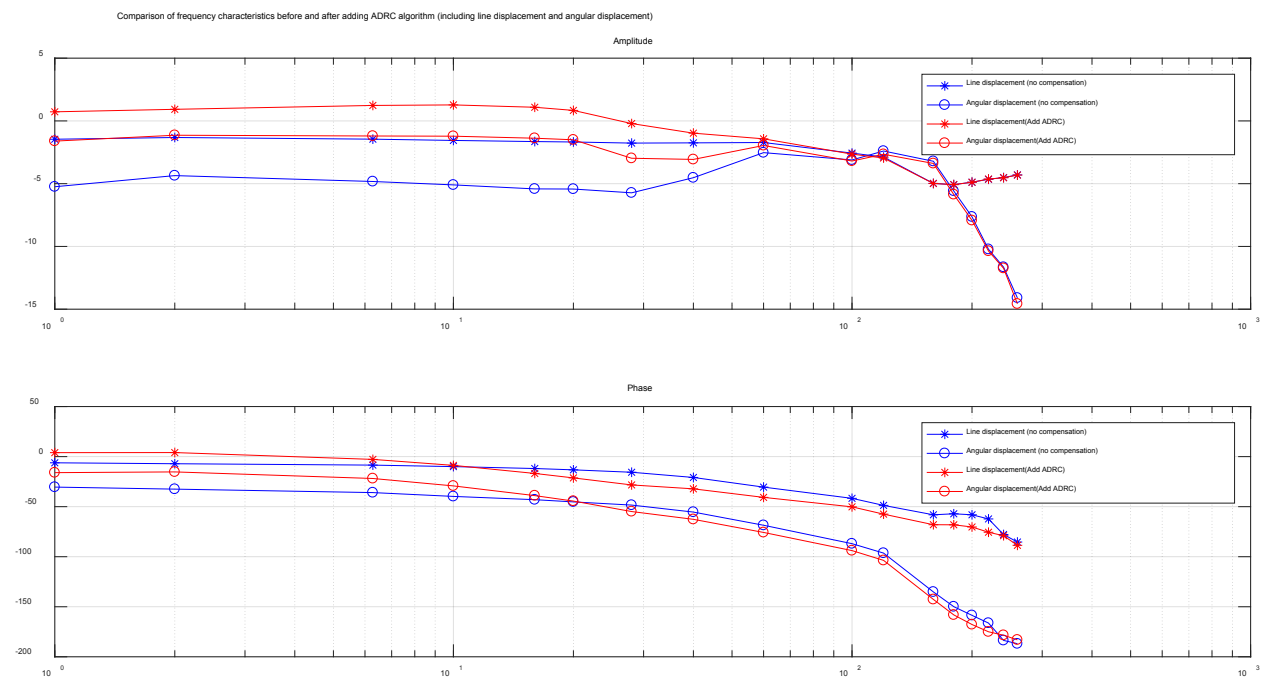

Fig. 7. Bode diagram curve comparison.

After adding the auto-disturbance algorithm, due to the introduction of the advance compensation amount, the line displacement will advance the control signal in the phase, thereby canceling the deformation caused by the coulomb friction torque of the nozzle, so that the angular displacement is close to the command and can satisfy design expectations.

\section{Concluding remarks}

In the classical PID closed-loop control electromechanical servo system, by introducing the auto disturbance rejection control algorithm, the problem of the characteristic deterioration of the bead nozzle or the ball and socket nozzle due to its own nonlinear Coulomb friction torque can be effectively compensated. Especially for the low frequency control signal, it has obvious effect on the improvement of the phase characteristics of the system. However, the study found that as the frequency of the control signal increases, the compensation effect will gradually become less obvious, and the algorithm can further optimize the design in order to obtain better control performance.

For the thrust vector control system, it is characterized by the sensitivity of the control characteristics of the middle and low frequency bands, and for the signals with higher frequencies, there is usually no strict characteristic requirement. Therefore, the nonlinear control algorithm based on the auto-disturbance control technology studied in this paper can better compensate the nonlinear load influence of the bead nozzle and the ball and socket nozzle. The algorithm has obvious effects on improving the overall performance of the thrust vector control and improving the overall control stability of the rocket.

\section{References}

1. Nicholas, T.and Rossi, R. US Missile Data Book [M]. Data Search Associates, 1999.

2. AIAA Aerospace Design Engineers Guide [M]. American Institute of Aeronautics and Astronautics. 1993.

3. Brebner, G.G. The Control of Missiles [M]. AGARD-LS-98, 1979. 
4. R.J.Zeamer and F.H.Woodberry. Solid Rocket Thrust Vector Control [M]. NASA SP-8114, 1974.

5. J.Han. Control theory: Model approach or control approach [J]. Syst. Sci. Math., 1989(9): 328-335.

6. J.Han. Active disturbances rejection control technique [J]. Frontier Science., 2007(1): 24-31.

7. Jingqing Han. Linearity and Nonlinearity in Feedback Systems [J]. Control and Decision, 1988(2):56-59.

8. D. Basmadian. Mathematical Modeling of Physical Systems [M]. New York: Oxford University Press, 2003.

9. F. Bullo, A.D. Lewis. Geometric Control of Mechanical Systems: Modeling, Analysis, and Design for Simple Mechanical Control Systems [M]. New York: Springer Verlag, 2004.

10. J.D. Anderson. Fundamentals of Aerodynamics [M]. New York: McGraw-Hill Inc., 2005.

11. J. Han. Auto-disturbances-rejection controller and its applications [J]. Trans. Control Dec., 1998(1): 19-23.

12. J.Q. Han. The extended state observer of a class of uncertain systems [J]. Control Decision, vol. 2009(10): 900-906.

13. J.Q. Han. From PID to active disturbance rejection control [J]. IEEE Trans. Ind. Electron., 1997. (56): 173-181. 